\title{
Probing Magnetic Mysteries with Stellar Flares
}

\author{
Rachel A. Osten \\ Space Telescope Science Institute, 3700 San Martin Drive, Baltimore, MD 21218, USA \\ email: osten@stsci.edu
}

Invited Talk

\begin{abstract}
Flares are a fact of life for stars in the cool half of the H-R diagram. The production of magnetic fields and the consequent dynamic interactions of field and plasma give rise to observational phenomena which span the electromagnetic spectrum. Stellar flares have an impact not only on the stellar atmosphere but also the stellar environment, which can include forming and already formed planets. This talk gave a brief review of our current state of understanding of stellar flares, highlighting some of the main unanswered questions by a panchromatic approach. It also emphasized commonalities between observations and analysis of stellar flares and other types of transient and variable sources.
\end{abstract}

\section{Microscopy of the Interstellar Medium}

\author{
Mark Walker \\ Manly Astrophysics, 3/22 Cliff St, Manly 2095, Australia \\ email: Mark. Walker@manlyastrophysics . org \\ Invited Talk
}

\begin{abstract}
Radio-wave scintillation arises from inhomogeneities in the ionised ISM. Only small columns of free-electrons are needed to introduce significant phase changes, and the relevant length scales (around the Fresnel scale) are tiny, making scintillation a sensitive technique for studying the ISM on small-scales. On these scales the ISM is surprisingly rich in features, being densely populated by compact structures which manifest high stresses. These structures appear to be an entirely new component of the Galaxy.
\end{abstract}

